\title{
Incorporación de mujeres indígenas mexicanas en la academia universitaria
}

\author{
Incorporation of Indigenous Women as Academics \\ in Two Mexican Public Universities. Experiences \\ from the Academic Profession
}

\author{
Norma Molina Fuentes \\ Universidad Tecnológica de México (unitec), México \\ norma8291@hotmail.com \\ norma_molina@my.unitec.edu.mx
}

\begin{abstract}
8
RESUMEN

El aumento, paulatino y lento, del número de mujeres indígenas en las instituciones de educación superior (IES) es una constante. Sin embargo, la literatura sobre estas mujeres es prácticamente inexistente. Por lo tanto, la presente investigación tiene como finalidad hacer una aportación al estudio sobre las académicas que se autoadscriben como parte de un pueblo indígena. Para tal propósito, se entrevistó a un total de 14 académicas en dos universidades ubicadas en estados con alta presencia de población indígena: Yucatán y Veracruz. Las universidades a las cuales pertenecen son la Universidad Autónoma de Yucatán (UADY) y la Universidad Veracruzana Intercultural (UVI). El objetivo fue identificar y explicar las principales problemáticas a las que se han enfrentado, desde la colonialidad del saber, en su intención de ejercer el oficio académico a partir de epistemologías y prácticas más incluyentes e interculturales.
\end{abstract}

Palabras clave: Indígenas; académicas; decolonialidad; educación superior.

\begin{abstract}
The gradual and slow increase in the number of indigenous women in Higher Education Institutions (HEI) is a constant. However, the literature on indigenous women entering university is virtually non-existent. Therefore, this research aims to contribute to the study of women academicians who self-identify as part of indigenous peoples. For this paper, a total of fourteen academicians were interviewed in two universities located in states with a high presence of indigenous population: Yucatan and Veracruz. The universities to which they belong are Universidad Autónoma de Yucatán (UADY) and Universidad Veracruzana Intercultural (UVI). The aim is to identify and explain the main problems faced by women academicians, since the coloniality of knowledge, in their in-
\end{abstract}

60 - Incorporación de mujeres indígenas mexicanas en la academia universitaria Norma Molina Fuentes. DIDAC 76 (2020): 60-68 
tention to exercise the academic profession based on epistemologies and more inclusive and intercultural practices.

Keywords: Indigenous; Women Academicians; Decoloniality; Higher Education.

Fecha de recepción: 25/10/2019

Fecha de aceptación: 28/01/2020

\section{Introducción}

El presente artículo forma parte de una investigación doctoral más amplia, la cual tuvo como objeto de análisis las trayectorias educativa y académica, así como el ingreso de un grupo de mujeres indígenas en relación con su condición étnica-lingüística y de género. Dicha investigación se aborda desde la perspectiva decolonial y de género. Para analizar el colonialismo interno de la sociedad mexicana se tomaron en consideración las condiciones socioeconómicas y las experiencias migratorias - de primera o segunda generación- de las entrevistadas.

Asimismo, recurriendo a la colonialidad del saber, se hizo visible la forma de conocimiento eurocéntrica y monolingüística, en español, bajo la cual fueron educadas. Igualmente, se analizaron las implicaciones de su condición étnica y lingüística en su paso por el sistema educativo, ingreso y trayectoria académica. A lo largo de este artículo se presentan sólo los hallazgos encontrados en el apartado sobre profesión académica e interculturalidad, en el cual se indaga el ejercicio de la profesión académica en relación con la adscripción étnica de las académicas.

Quijano (2007) define la colonialidad como un patrón mundial de dominación dentro del modelo capitalista, fundado en una clasificación racial y étnica de la población del planeta que opera en distintos ámbitos. Según el autor, la colonialidad es una estructura de dominación y explotación que se inicia con el colonialismo, pero que se extiende hasta hoy día como su secuela. En otras palabras: "con América (Latina) el capitalismo se hace mundial, eurocentrado y la colonialidad y la modernidad se instalan, hasta hoy, como los ejes constitutivos de este particular modelo de poder" (Quijano, 2007, p. 91).

Aunado a la colonialidad se desprende la colonialidad del saber, entendida como el posicionamiento del eurocentrismo en tanto perspectiva única del conocimiento, concepto acuńado por Lander (2000). Este posicionamiento, plantea Walsh (2008), es particularmente evidente en los sistemas educativos donde se elevan el conocimiento y la ciencia europea como los marcos científico-académico-intelectuales. Otro autor que apela a la diversificación del conocimiento es Santos (2011), quien plantea que la idea de que exista un solo conocimiento válido es sumamente injusta y antidemocrática. Más aún, según Castro (2005), la universidad representa esencialmente la hybris del punto cero, es decir, un lugar donde se materializa la geopolítica del conocimiento.

Con base en esta línea de pensamiento, se aborda la función social de las universidades referidas. Por un lado, las universidades interculturales (UI) son en gran medida una respuesta a exigencias sociales de la población indígena que ha sido históricamente excluida en la educación terciaria. Primero, porque los jóvenes no lograban acceder a ésta, y segundo, porque su conocimiento quedó, en gran parte, relegado del currículo educativo. Por otro lado, algunas instituciones de educación superior (IES) de corte tradicional, como la Universidad Autónoma de Yucatán (UADY) —ubicadas en zonas con alta presencia de población indígena-, han tenido que implementar acciones para responder a demandas específicas de su población con adscripción étnica. Por ello, Walsh (2006) afirma que la universidad ha sido una institución que ilustra claramente las tensiones y pugnas entre seres y saberes, poderes y geopoderes, y en la que se crean circunstancias complejas.

Es desde este marco de referencia, que la presente investigación pretende dar respuesta a la siguiente interrogante: ¿cuáles han sido las problemáticas a las que se han enfrentado las académicas indígenas desde la colonialidad del saber, en su búsqueda del ejercicio de una profesión académica más incluyente e 
intercultural? Así, a lo largo de este escrito se exponen los hallazgos de uno de los apartados de la tesis, el cual mostró cómo es que más allá del modelo educativo de cada IEs, las académicas se han enfrentado a resistencias propias del habitus y ethos académico, en su intención de ejercer su profesión desde una epistemología más incluyente e intercultural, que abarque otro tipo de saberes y prácticas académicas.

¿Quiénes son las académicas indígenas?

Conocer los orígenes de las académicas permitió entender, entre otros temas, cómo se han fraguado sus historias, su paso por la escolaridad formal —las principales problemáticas que enfrentaron-, las consecuencias de la migración, fuera de sus comunidades, por ejemplo, su relación con el hecho de hablar o no la lengua, así como los motivos de sus padres para trasmitírselas o no. Sintetizando lo anterior: ocho de las entrevistadas son originarias de Yucatán, tres de Veracruz y una más de Querétaro. La mayoría, 10 de ellas, son la primera generación en migrar de sus pueblos de origen; tres más, la segunda. Nueve de ellas son hablantes de sus lenguas maternas, dos más la entienden, pero no la hablan, y tres no entienden ni hablan la lengua de sus padres. La Tabla 1 muestra una síntesis de esos rasgos.

TABLA 1

Datos generales de las académicas indígenas

\begin{tabular}{|c|c|c|c|c|c|c|}
\hline & ETNIA & LENGUA & EDAD & $\begin{array}{l}\text { MIGRACIÓN } \\
\text { GENERACIÓN }\end{array}$ & EsTADo CIVIL & Hijos (As) \\
\hline \multirow{6}{*}{5} & náhuatl & entiende/habla & 31 & primera & Unión libre & No \\
\hline & náhuatl & entiende/habla & 28 & no migró & Soltera & No \\
\hline & zapoteca & entiende/no habla & 34 & primera & Soltera & No \\
\hline & totonaco & entiende/habla & 37 & primera & Casada & 1 \\
\hline & popoluca & entiende/habla & 26 & primera & Soltera & No \\
\hline & กีañuu & entiende/habla & 35 & primera & Casada & 2 \\
\hline \multirow{8}{*}{ 空 } & maya & no entiende/no habla & 38 & segunda & Casada & No \\
\hline & maya & no entiende/no habla & 41 & segunda & Soltera & No \\
\hline & maya & no entiende/no habla & 40 & segunda & Casada & No \\
\hline & maya & entiende/habla & 54 & primera & Casada & No \\
\hline & maya & entiende/no habla & 53 & primera & Soltera & No \\
\hline & maya & entiende/habla & 73 & primera & Soltera & No \\
\hline & maya & entiende/habla & 28 & primera & Soltera & No \\
\hline & maya & entiende/habla & 58 & primera & Casada & 1 \\
\hline
\end{tabular}

Los grados alcanzados por las académicas hablan de un éxito escolar. Cinco de ellas cuentan con estudios de doctorado - algunas estaban en proceso de titulación-; ocho con grado de maestría y dos con licenciatura. Cuando se hace referencia a un éxito escolar se concibe en relación con los grados académicos alcanzados en comparación no sólo a la población indígena, sino en general, para quienes el promedio de escolaridad es de 9.1 años, según el Instituto Nacional de Estadística y Geografía (INEGI) (2015).
El tema migratorio ligado a la escolaridad es una constante encontrada en los estudios sobre profesionistas, líderes políticos e intelectuales de condición indígena. Oehmichen (1999) señala que las migraciones de las mujeres se encuentran más condicionadas que las de los varones por la etapa en su trayectoria de vida, por su posición en el hogar, por su estado civil, por la presencia de pareja e hijos y por la estructura familiar. Así, el primer filtro que sortearon algunas de ellas fue contar con la aprobación familiar para irse de casa, con la finalidad de 
continuar sus estudios. El segundo fue enfrentarse a las problemáticas de corte económico para sostenerse fuera de la economía familiar.

Hablar o no hablar una lengua indígena en México sigue teniendo repercusiones diferentes. La perspectiva decolonial nos dice que una de las formas más explícitas de la colonialidad se da a través de la primacía de una (o unas) lengua (s) sobre las otras. El no hablar ni entender la lengua se puede explicar, como lo argumenta Aguilar (2015), por la discriminación sistemática que, por siglos, ha padecido la población indígena. Si bien México es un país plurilingüe, no es posible considerarlo como multilingüe, dadas las relaciones asimétricas de poder que están presentes en todos los espacios sociales.

Ahora bien, respecto a los oficios y las profesiones de los padres de las académicas, sabemos que algunos de ellos se desempeñaron como comerciantes, albañiles, campesinos y jornaleros. Sólo dos de ellos contaban con estudios profesionales: uno era maestro de educación primaria, y el otro, abogado. Por su parte, las madres ejercían los oficios de comerciantes, campesinas, trabajadoras del hogar y empleadas.

Las historias de vida de las académicas coinciden en algunos aspectos y difieren en otros. Sin embargo, una constante se repite: desde la educación bási- ca sobresalieron como estudiantes de excelencia, con los mejores promedios de sus clases. A la academia, llegaron las mujeres que lograron sobrevivir al cuello de botella - proceso en el cual la población estudiantil queda excluida del sistema educativo conforme avanzan los grados escolares-, que aplica para todas las niñas y las jóvenes de este país, y cuyo efecto se acentúa aún más en la población indígena.

\section{Etnicidad y profesión académica}

\section{Profesión académica y etnicidad desde la Universidad} Autónoma de Yucatán (UADY)

La Universidad Autónoma de Yucatán (UADY) tiene su origen en 1922 cuando se creó la llamada Universidad Nacional del Sureste. A pesar de estar ubicada en un área geográfica con alta población indígena, fue hasta el año 2010 cuando implementó un programa de atención a la comunidad estudiantil maya. Asimismo, incorporó saberes de la cultura maya en su currículo formal como materia transversal en todos sus programas de estudio.

Lander (2000) nos dice que, en el proceso histórico de la constitución de las ciencias sociales, las otras formas de ser, de organización y del saber de las sociedades fueron transformadas no sólo en "diferentes", sino además en "carentes”, "arcaicas",

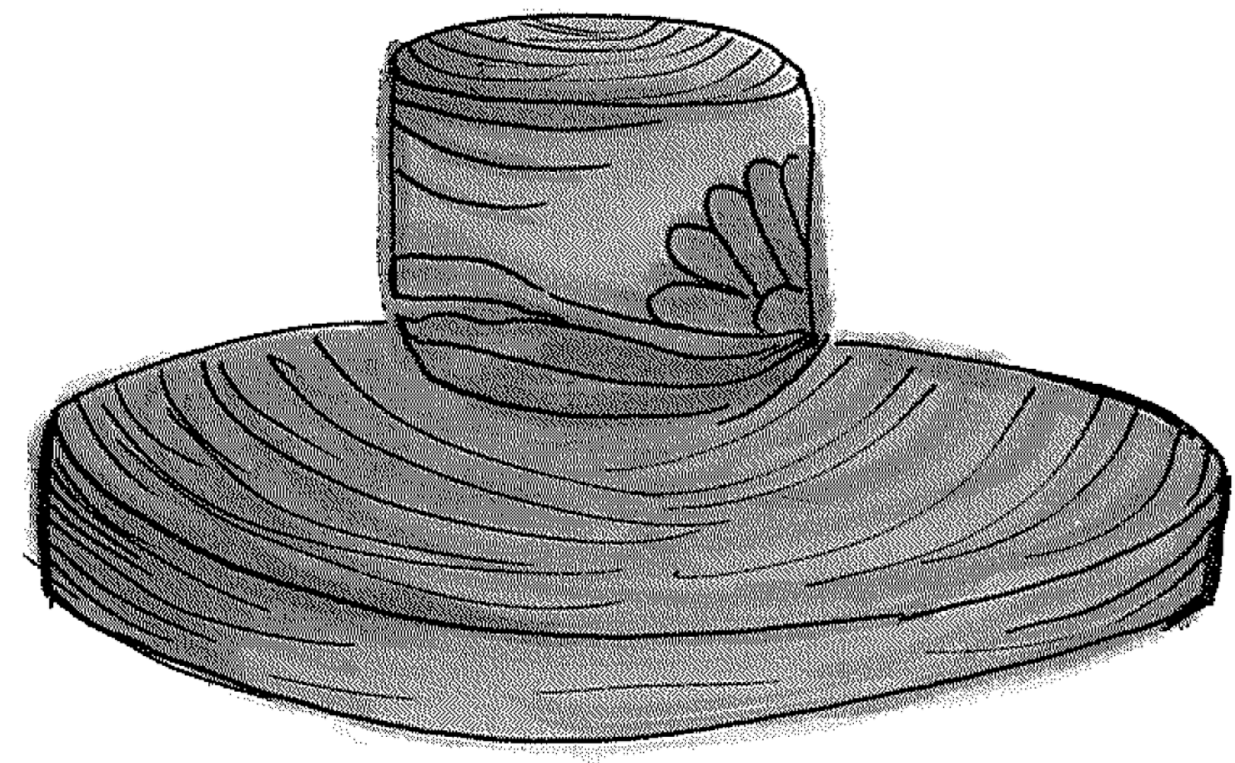

Incorporación de mujeres indígenas mexicanas en la academia universitaria $\bullet 63$ Norma Molina Fuentes. DIDAC 76 (2020): 60-68 
"primitivas", "tradicionales" y "premodernas". Dicho postulado se confirma en la narración de las experiencias que comparten las académicas de la UADY, en las cuales ponen a discusión la colonialidad del saber que han experimentado en su ejercicio académico.

Una de las entrevistadas relata las dificultades epistemológicas que le representó la decisión de hacer su trabajo de investigación en su propio pueblo. Desde la antropología, situarse como "los otros" o "las otras" le resultó un proceso complicado, más aún verlo desde lo exótico (como ella percibía que se les veía). A esto, se le sumaron las problemáticas de la interacción en su rol de investigadora y pertenecer a la comunidad. Por ejemplo, el hecho de ser mujer y que la vieran acompañada constantemente por colegas varones fue objeto de críticas por la gente de su pueblo, en cuanto a su moralidad y sexualidad. Otro aspecto comentado por la entrevistada fue su postura contra las políticas y los proyectos que implementaba el, ahora extinto, Instituto $\mathrm{Na}$ cional Indigenista (INI), organismo que pretendía hacer videos sobre las comunidades desde la otredad, presentando — de nuevo- lo diferente como exótico, ante lo cual proponía capacitar personas para que las comunidades se autofilmaran.

Para poder enmarcar lo narrado por las entrevistadas, respecto a sus experiencias académicas en el campo universitario, se retoma la teoría de campo de Bourdieu (2002, 2008), quien plantea que, dado que en el campo académico están en disputa los lugares de pertenencia y los criterios de permanencia, los sujetos se afrontan en la lucha simbólica por el monopolio legítimo del campo. El campo académico tiene sus propias lógicas, habitus y estructuras que organizan y norman a las instituciones de educación superior. Y aunque tanto la UADY como la Universidad Veracruzana Intercultural (Uvi) están creadas bajo modelos diferentes y con características particulares, ambas se guían por el ethos académico que rige a las instituciones de educación superior.

Una de las académicas que se integró a la UADY a partir de la creación de los proyectos de inclusión e interculturalidad puestos en marcha en 2010, narra que desde su experiencia la interculturalidad como tal no existe como en dichos programas, pues ha visto, en más de una ocasión, la reproducción de prácticas indigenistas. No obstante, considera su trabajo como resultado de un compromiso de carácter étnico y una contribución al reconocimiento de lo que ella considera el pensamiento de otra sociedad: la maya. En esta lógica, nos dice Walsh, "el uso intencional de interculturalidad es una parte constitutiva de las estrategias del Estado que refleja un esfuerzo por incorporar las demandas y los discursos subalternos 'dentro' del aparato estatal' (2006, p. 44).

Otra de las académicas nos dice textualmente:

ahorita estoy en riesgo de perder el $\mathrm{sNI},{ }^{1}$ revisa mi página para que veas todo el tipo de actividades que hago con la gente, con las comunidades, eso no vale, para mis pares yo no estoy haciendo nada, mi trabajo no está dando luz a ellos, los otros "sabios" (fragmento de entrevista).

Como muestran Arzate y Romero (2007), en las últimas décadas las universidades han estado regidas por un modelo neoliberal que distribuye los recursos mediante una lógica de eficiencia medida por la evaluación. Bajo esta lógica, actividades que las académicas consideran prioritarias, como el trabajo en comunidad y la enseñanza de la lengua, quedan fuera de las que, en el campo universitario, son consideradas valiosas y evaluables. Así, el campo universitario, se ve rebasado por acciones como las que realizan las académicas. La estructura de dicho campo no contempla como importante o "redituable" la vinculación con los pueblos y las comunidades.

Este mismo fenómeno que describe la académica lo han abordado Pérez y Botero (2013), quienes en su investigación muestran que, en general, las científicas afrocolombianas ubicadas en universidades regionales reconocen que ocupan una posición marginal o no privilegiada dentro del campo académico. Aquellas que priorizan el trabajo étnico y co-

\footnotetext{
${ }^{1}$ Sistema Nacional de Investigadores
}

64 - Incorporación de mujeres indígenas mexicanas en la academia universitaria Norma Molina Fuentes. DIDAC 76 (2020): 60-68 
munitario saben que mucho de esa labor no tendrá una repercusión directa en el campo universitario, dado que la obtención del prestigio y del reconocimiento pasa por otras vías y no son siempre en consonancia con las dinámicas de un campo académico local, sino que están internacionalizadas en sus circuitos de producción y de difusión.

No obstante, también hay que mencionar que los intereses de las académicas no son homogéneos en el grupo de la UADY. Algunas ejercen su quehacer científico sin que esté ligado a temas de carácter étnico; por ejemplo, aquellas que están en el área de educación y en matemáticas no tienen líneas de investigación vinculadas con dichas preocupaciones, aunque a veces tocan el tema étnico, tangencialmente con sus alumnos.

Profesión académica y etnicidad desde la Universidad Veracruzana Intercultural (UVI)

Hacer un breve acercamiento a la interacción entre las académicas, la uvi y la comunidad conduce a aludir someramente la particularidad del modelo educativo de dicha universidad. La uvi imparte la licenciatura en Gestión Intercultural para el Desarrollo en sus cuatro regiones-sedes, que responde a los requisitos de inter o transdisciplinariedad, multimodalidad, flexibilidad curricular y autonomía estudiantil, mismo que ha adoptado la Universidad Veracruzana (Uv) en su conjunto, como su Modelo Educativo Integral y Flexible. Como todo proyecto de esta naturaleza, la UVI se ha enfrentado, en su etapa de consolidación, con diferentes problemas de índole económica, organizativa, epistemológica y política.

Una de sus problemáticas es que al ser una IEs de reciente creación y de corte intercultural se enfrenta a la desconfianza de los alumnos que prefieren inscribirse en universidades de corte tradicional. Dietz (2009) y Mateos y Dietz (2015) han realizado diversos estudios sobre la uvi para analizar la participación de los profesionistas indígenas y no indígenas, así como sus repercusiones en el tránsito, aún reciente, hacia el reconocimiento social, político e incluso jurídico de la comunidad en el ámbito de las universidades públicas. Una de las principales características de las uI es su claro objetivo de trabajar en coordinación con las comunidades de la región. Sin duda, esta intención explícita marca una diferencia con las universidades tradicionales.

Retomando las reflexiones de Villaseñor (2003) y Santos (2007) sobre la función social de la universidad, la uvi confronta la complicada tarea de proponer un currículo de corte intercultural, pero también la de incluir a otros actores dentro de los consejos formales de la institución, ${ }^{2}$ con la finalidad de ser una IEs verdaderamente incluyente. Una de las académicas nos dice "yo veo impactos en la formación de los jóvenes ya en el camino, ya en la práctica profesional, en el servicio en la comunidad (...) si bien ha tenido sus críticas y todo, para mí sigue siendo un programa bondadoso" (fragmento de entrevista). Para ellas, el vínculo y el trabajo con las comunidades representan una oportunidad de emprender proyectos conjuntos, pero también dificultades de diferente índole.

En sus investigaciones, Dietz (2008, 2009) muestra cómo los diferentes integrantes de la UVI diseñaron y proyectaron mecanismos de vinculación con las comunidades. Por un lado, recurrieron al habitus centrado en la praxis cultural y la identidad étnica, conforme con lo establecido por Bourdieu. Por el otro, al comunalismo, entendido como un modelo normativo-reivindicativo de "hacer comunidad" (Pérez, 2005), y cuyo objetivo es fortalecer y privilegiar los recursos endógenos tanto organizativos como simbólicos, tanto políticos como pedagógicos, frente a la imposición de modelos exógenos y colonizadores. Desde esta postura, arrancaron un trabajo con las comunidades a través del reconocimiento de su heterogeneidad, más allá de posturas esencializantes.

Sin embargo, mientras que la uvi cuenta con un fuerte apoyo en el conjunto de las sociedades regio-

\footnotetext{
${ }^{2}$ Aparte de la estructura orgánica convencional de toma de decisiones académica (consejo técnico y junta académica), la Universidad Veracruzana Intercultural (UVI) dispone de Consejos Consultivos, que — con presencia de las comunidades indígenas — vigilan, supervisan y asesoran el transcurso de los programas educativos, las titulaciones y las actividades de docencia, investigación y vinculación que se realizan desde la sede central y las sedes regionales (Dietz, 2009, p. 63).
} 
nales que atiende, al interior de la misma universidad persisten resistencias e incomprensiones. $\mathrm{Al}$ tratarse de una noción heterodoxa de "universidad", de "licenciatura" y de "plan de estudios", algunos sectores más tradicionales y "disciplinarios" de la academia pretenden relegar esta iniciativa a actividades no estrictamente docentes o investigadoras, sino a un "extensionismo" asistencialista de viejo cuño.

\section{Anotaciones finales}

Como se muestra, algunas de las académicas que se reconocen como mayas han cuestionado desde sus disciplinas la forma en que se ha llevado a cabo la investigación sobre los pueblos indígenas. Desde su postura, como académicas buscan otras formas de hacer investigación e ir más allá de mostrar a las comunidades como "los otros" de los cuales ellas son parte. Reconocen que hay un discurso de falsa interculturalidad, en el cual nuevamente los pueblos tienen que ser los interculturales, no así la demás población. Externan sus dudas acerca de enseñar el idioma maya, ya que la lengua puede ser una "arma de dos filos" (como una de ellas lo dice), dado que, probablemente, los investigadores que se acercan a las comunidades reproducirán esta visión colonizadora.

También, hacen una crítica a la falta de mecanismos en las universidades tradicionales para estrechar sus vínculos con la sociedad, en este caso, con las comunidades mayas. La lógica propia del campo universitario no tiene un rubro que visibilice y -en esta cultura de la evaluación constante por medio de los puntos- promueva la investigación o el trabajo comunitario. Así, algunas de ellas prefieren no buscar el reconocimiento e ingreso que otorga el Sistema Nacional de Investigadores (sNI), por ejemplo, para tener el tiempo de continuar con sus proyectos en las comunidades.

Otras más, para quienes la investigación étnica no es central en su trabajo, tocan la temática étnica de manera tangencial, pero no menos significativa. Por ejemplo, mostrarse como maya hablantes y visibilizar en el aula a los alumnos maya hablantes como

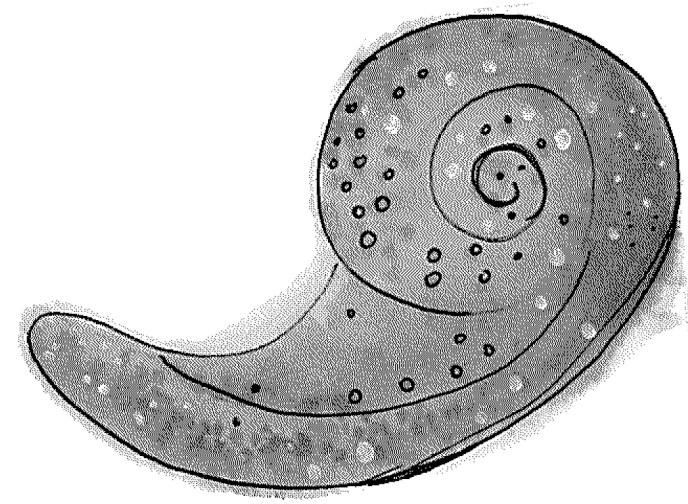

una forma de reconocimiento, esto con la finalidad de contrarrestar el racismo lingüístico que persiste. Para ellas, reconocerse frente a sus alumnos como maya hablantes fue un acto político y reivindicativo. Si bien la UADY tiene un programa que pretende impartir la materia de cultura maya en todas sus carreras, el estudio nos deja ver que son los sujetos, en este caso las académicas, los que desde su oficio diario pueden incidir en el tema reivindicativo.

Referirse a la misma tríada — universidad-académicas-comunidad-, desde el modelo de la uvi, lleva a hacer el reconocimiento y el cuestionamiento al modelo intercultural. Sectores críticos a las uI han visto a estas instituciones como apropiación, por parte de las instancias gubernamentales, de demandas legítimas de los pueblos indígenas. Esto llevó a la creación de universidades con una oferta educativa que ha sido fuertemente cuestionada, así como lo han sido sus medidas estratégicas para evitar la politización de este tipo de escuelas. Numerosas han sido las noticias acerca de las problemáticas políticas e imposiciones de rectores que realizan los gobernadores de los estados, haciendo de las uI botines políticos. Gómez (2016), en su estudio sobre la uvi, identifica la ambivalencia con la que nace la universidad: tiene que configurar el prestigio que acompaña a la "universidad", es decir, mostrar que es una IEs confiable; sin embargo, al estar ubicada en una zona indígena $\mathrm{e}$ identificarse como intercultural se le asocia fácilmente como una universidad de poca calidad.

Un punto no menos importante son las resistencias epistemológicas que las académicas encuentran dentro de la universidad: aunque la uvi se crea bajo

66 - Incorporación de mujeres indígenas mexicanas en la academia universitaria Norma Molina Fuentes. DIDAC 76 (2020): 60-68 
el modelo intercultural, finalmente son los sujetos quienes hacen las prácticas académicas, docentes que fueron formados en IEs tradicionales, reproduciendo así lo aprendido desde sus áreas de conocimiento o poniendo resistencia a la integración de otros tipos de saberes, por ejemplo. No obstante, reconocen los esfuerzos de múltiples actores por hacer funcionar y potenciar estas universidades en pro de los estudiantes y de las comunidades indígenas.
Para las académicas, ser parte de la universidad intercultural representa enfrentarse a las complicaciones y limitaciones de un proyecto nuevo $y$, a nivel personal, confrontar una incertidumbre laboral en su ejercicio profesional. Sin embargo, también valoran estar en una universidad de corte intercultural, formar jóvenes de las regiones, hacer trabajo comunitario y, sobre todo, investigar con y acerca de los pueblos indígenas. ID

\section{REFERENCIAS}

Aguilar, Y. (1 de agosto de 2015). ¿Es México un país multilingüe? Este Pais. Recuperado de https://archivo.estepais.com/ site/2015/es-mexico-un-pais-multilingue/

Arzate, J. \& Romero, J. (2007). Diversificación, crecimiento y desigualdad en la educación superior: la dimensión relativa de la universidad pública en México. Tiempo de Educar, 8(16), 277-303.

Bourdieu, P. (2002). Campo de poder, campo intelectual. Argentina: Montressor.

Bourdieu, P. (2008). Homo academicus. Argentina: Siglo xxI Editores.

Castro, S. (2005). La hybris del punto cero. Ciencia, raza e Ilustración en la Nueva Granada (1750-1816). Bogotá: Pontificia Universidad Javeriana.

Dietz, G. (2008). La experiencia de la Universidad Veracruzana Intercultural. En D. Mato (Coord.), Diversidad cultural e interculturalidad en educación superior. Experiencias en América Latina (pp. 359-370). Venezuela: Unesco-IESALC.

Dietz, G. (2009). Los actores indígenas ante la "interculturalización” de la educación superior en México: ¿empoderamiento o neoindigenismo? Revista Latinoamericana de Educación Inclusiva, 3(2), 55-75.

Gómez, D. (2016). Herencias, contradicciones y resignificaciones de la politica educativa intercultural en el sur de Veracruz, México. Un estudio acerca de la Universidad Veracruzana Intercultural (UVI) (Tesis doctoral). Universidad Complutense de Madrid, Madrid.

Instituto Nacional de Estadística y Geografía (INEGI). (2015). Educación. Recuperado de http://cuentame.inegi.org.mx/ monografias/informacion/mex/poblacion/educacion.aspx?tema $=$ me\&e $=15$

Lander, E. (2000). Ciencias sociales: saberes coloniales y eurocéntricos. En E. Lander (Ed.), La colonialidad del saber: eurocentrismo y ciencias sociales. Perspectivas latinoamericanas (pp. 11-39). Buenos Aires: CLACso.

Mateos, L. \& Dietz, G. (2015). ¿Qué de intercultural tiene la "universidad intercultural"? Del debate político-pedagógi- co a un estudio de caso veracruzano. Relaciones. Estudios de Historia y Sociedad, 141, 13-45.

Oehmichen, C. (1999). La relación etnia-género en la migración femenina rural-urbana: mazahuas en la Ciudad de México. Iztapalapa: Revista de Ciencias Sociales y Humanidades, 45, 107-132.

Pérez, T. \& Botero, D. (2013). Entre el afuera y el adentro. La configuración del campo académico y sus fronteras desde las prácticas comunicativas de científicas negras en Colombia. Co-Herencia, 10(18), 189-220.

Pérez, M. (2005). La comunidad indígena contemporánea. Límites, fronteras y relaciones interétnicas. En M. Lisbona (Coord.), La comunidad a debate. Reflexiones sobre el concepto de comunidad en el México contemporáneo (pp. 87-100). Michoacán-Chiapas, México: El Colegio de Michoacán-Universidad de Ciencias y Artes de Chiapas.

Quijano, A. (2007). Colonialidad del poder y clasificación social. En S. Castro \& R. Grosfoguel (Comps.), El giro decolonial. Reflexiones para una diversidad epistémica más allá del capitalismo global (pp. 93-126). Bogotá: Siglo del Hombre Editores.

Santos. B. (2007). La universidad en el siglo XXI. Para una reforma democrática y emancipadora de la universidad. Bolivia: Cides-umsa.

Santos, B. (2011). Introducción: las epistemologías del Sur. En СІDов (Org.), Formas-Otras. Saber, nombrar, narrar, hacer (pp. 9-22). Barcelona: сіров Ediciones.

Villaseñor, G. (2003). La función social de la educación superior en México. La que es y la que queremos que sea. México: UAM/ CESU-UNAM/UV.

Walsh, C. (2006). Interculturalidad y colonialidad del saber. Un pensamiento y posicionamiento otro desde la diferencia colonial. En C. Walsh, W. Mignolo \& A. García (Comps.), Interculturalidad, descolonización del Estado y del conocimiento (pp. 21-70). Buenos Aires: Ediciones del Signo.

Walsh, C. (2008). Interculturalidad, plurinacionalidad y decolonialidad: las insurgencias político-epistémicas de refundar el Estado. Tabula Rasa, 9, 131-152. 
Aguilar, Y. (10 de septiembre de 2015). ¿Para qué publicar libros en lenguas indígenas si nadie los lee? Este País. Recuperado de https://archivo.estepais.com/site/2014/ para-que-publicar-libros-en-lenguas-indigenas-si-nadielos-lee/

Alvarado, J. (2016). Pensar la universidad en perspectiva decolonial. Revista de Filosofía, 84(3), 116-128.

Blazquez, N. (2011). El retorno de las brujas. Incorporación, aportaciones y críticas de las mujeres a la ciencia. México: CEIICH-UNAM.

Cabnal, L. (2010). Acercamiento a la construcción de la propuesta de pensamiento epistémico de las mujeres indígenas feministas comunitarias de Abya Yala. En Feminismos diversos: el feminismo comunitario. Madrid: ACsUR-Las Segovias, Asociación para la Cooperación con el Sur.

Casillas, M. (1987). Notas sobre el proceso de transición de la universidad tradicional a la moderna. Los casos de la expansión institucional y la masificación. Sociológica. Revista del Departamento de Sociología, 5(2).

Cumes, A. (2014) Esencialismos estratégicos y discursos de descolonización. En M. Millán (Coord.), Más allá del feminismo: caminos para andar. México: Red de Feminismos Descoloniales.
Hall, S. (1996). Introducción: ¿quién necesita “identidad”? En S. Hall \& P. Guy (Comps.), Cuestiones de identidad cultural (pp. 13-39). Buenos Aires-Madrid: Amorrortu editores.

Lugones, M. (2014). Colonialidad y género: hacia un feminismo descolonial. En W. Mignolo (Comp.), Género y descolonialidad (pp. 13-41). Buenos Aires: Ediciones del Signo.

Molina, N. (2016). Inserción laboral en espacios académicos de intelectuales indígenas en México. Ex becarios del International Fellowships Program. Revista Iberoamericana de Educación, 72(1), 69-88.

Molina, N. (2019). Arando surcos: incorporación de las mujeres indígenas como académicas en dos universidades públicas mexicanas. Universidades, 70(81), 11-22.

Quijano, A. (1992). "Raza”, “etnia” y "nación” en Mariátegui. Cuestiones abiertas. Estudios Latinoamericanos, 2(3), 3-19.

Schmelkes, S. (2008). Creación y desarrollo inicial de las universidades interculturales en México: problemas, oportunidades, retos. En D. Mato (Coord.), Diversidad cultural e interculturalidad en educación superior. Experiencias en América Latina (pp. 359-370). Venezuela: Unesco-IESALC.

Valladares, L. (2004). Mujeres ejemplares: indígenas en los espacios públicos. ALTERIDADES, 14(28), 127-147.

Zapata, C. (2008). Los intelectuales indígenas y el pensamiento anticolonialista. Discursos/prácticas, 2(1), 113-140.

\section{Semblanza}

Doctora y maestra en Ciencias con especialidad en Investigación Educativa por el Centro de Investigación y de Estudios Avanzados (Cinvestav) del Instituto Politécnico Nacional (IPN).

Licenciada y especialista en Sociología de la Educación Superior por la Universidad

Autónoma Metropolitana (UAM). Sus temas de investigación son: educación superior, género e interculturalidad. Integrante de la Red sobre Internacionalización y Movilidades Académicas y Científicas (RIMAC). Docente de instituciones de educación superior (IES) de carácter privado. 